\title{
Plant Pangenomics: Approaches, Applications and Advancements.
}

Monica Furaste Danilevicz ${ }^{1}$, Cassandria Geraldine Tay Fernandez ${ }^{1}$, Jacob Ian Marsh ${ }^{1}$, Philipp Emanuel Bayer ${ }^{1}$, David Edwards ${ }^{1,2}$

${ }^{1}$ School of Biological Sciences and Institute of Agriculture, University of Western Australia, Perth, WA, Australia

${ }^{2}$ Correspondent author: dave.edwards@uwa.edu.au ; School of Biological Sciences and Institute of Agriculture, (M084) University of Western Australia, Perth, WA, Australia

\begin{abstract}
With the assembly of increasing numbers of plant genomes, it is becoming accepted that a single reference assembly does not reflect the gene diversity of a species. The production of pangenomes, which reflect the structural variation and polymorphisms in genomes, enables in depth comparisons of variation within species or higher taxonomic groups. In this review, we discuss the current and emerging approaches for pangenome assembly, analysis and visualisation. In addition, we consider the potential of pangenomes for applied crop improvement, evolutionary and biodiversity studies. To fully exploit the value of pangenomes it is important to integrate broad information such as phenotypic, environmental, and expression data to gain insights into the role of variable regions within genomes.
\end{abstract}

Keywords: pangenome assembly, variation graphs, pangenome visualisation, crop improvement, genomic diversity.

\section{General introduction}

Plants have dynamic genomes, often with a history of multiple rounds of whole genome duplication and subsequent fractionation. Other mechanisms also play an important role in plant genome variation such as gene tandem duplications, transposable element activity, deletions, genome rearrangement and recombination across populations [1-6]. These processes can lead to gene presence/absence variation (PAV) and structural variation (SV), which is associated with phenotypic diversity, adaptation and selection. Because of this genomic variation, single reference genome assemblies do not reflect the genomic diversity within a species, and so a pangenome is required to capture this diversity. A pangenome reference combines nonredundant genome sequences into a single annotated file, and consists of core and dispensable 
regions. The core is composed of sequences present in all individuals, while dispensable regions are present only in a subset of the individuals. Advances in DNA sequencing have enabled the assembly of increasing numbers of genomes and pangenomes, and as genome sequencing continues to advance, there are opportunities to apply pangenome assembly and analysis to explore adaptation and evolution, both within and across species, gaining insights into the function of genomes with applications for crop breeding.

\section{Approaches for pangenome assembly}

Genome and pangenome assembly in plants is often challenging due to the presence of a large numbers of repeats and multiple rounds of polyploidy [7, 8]. The traditional de novo assembly and comparison approach (Figure 1A; [9-12]) was first used to demonstrate significant genomic differences between individuals. It has the benefit of providing the physical position of genes and other genomic elements, though is relatively costly, and assembly errors or variation in annotations often confound the identification of true genomic differences between individuals [13]. In contrast, the iterative mapping and assembly approach [13-17] involves starting with a single reference genome and sequentially updating it with non-redundant sequences from other individuals to build a pangenome (Figure 1B; [14]). This approach permits the pooling of large numbers of individuals with lower sequencing coverage than required for multiple whole genome assemblies, with subsequent calling of PAV by remapping reads back to the pangenome. However, the iterative approach struggles to assemble sequences that are highly repetitive or characterise large SVs which cannot be spanned by single reads [18]. The benefits and limitations of these two methods makes them highly complementary. The combination of a small number of high quality de novo assemblies with a large number of individuals sequences with $>10 x$ coverage permits the physical placement of variable genes, the capture of rare genes, as well as an assessment of gene PAV across a large population [19].

The majority of plant pangenomes to date have been produced using Illumina short read data, predominantly due to the low error rate $(<1 \%,[20])$ and relatively low cost per Gbp. However, the rapid advancements made in long and ultra-long read sequencing technology are likely to lead to a significant change in how pangenomes are constructed. Long read sequencing permits the spanning and resolution of complex repetitive regions and can also be used to characterise large SVs (>500bp) [21], although long reads generally have a higher error rate [22]. A hybrid approach using short and long reads has been shown to overcome the drawbacks of using either data type alone and successfully produced contiguous plant genome assemblies, 
including the Solanum verrucosum and Solanum pennelli genome assemblies [22, 23]. More recently, bi-directed variation graphs (VG) have been used for pangenome construction. VG uses sequencing reads supported by linear genome references to produce a graph representation of the variable regions, avoiding assembly bias towards a reference genome (Figure 1A; [24, 25]). VG tools are fast and robust assemblers that can resolve complex structural variants more effectively than linear assemblers, as the location of variants are more widely represented by the set of graph paths (Figure 1C). VG enables a population-scale representation of the genome, allowing for the continuous addition of novel sequencing data [25-27]. Variation Graph and Graph Genome are two of the latest toolkits able to construct, manipulate, and perform variant calling in human sized genomes, presenting a modest gain in SNP calling accuracy compared linear analysis tools [24, 25]. Currently, VG applications in plant pangenomes have been limited by high structural complexity and repetitiveness of some plant genomes. There is also a scarcity of tools available to perform common downstream analyses and visualisation of the graph genomes. However, considering the advantages provided by graph pangenomes and the latest advances in the development of VG tools for the construction of the human pangenome, it is likely that these constraints will be addressed in the future.

\section{Deep learning tools for pangenome assembly and analysis}

Machine learning is being increasingly applied to plant genome analysis [28-31], and has assisted in the identification of sequence tags for use as genetic anchors for maize pangenome assembly [8, 32]. Machine learning approaches generally rely on domain-specific knowledge to be hard coded, such as an explicit description of the target genomic region, which requires the operator to have extensive knowledge of the field [33]. In contrast, deep learning (DL) models can autonomously detect and learn from patterns in example data with minimal user input in regards to data description, but still require data pre-processing and coding expertise in order to build an appropriate architecture to solve the problem [34]. DL has the potential to leverage large and complex data sets, generating accurate classification, prediction or clustering outputs based on a wide range of statistical methods (Figure 2).

Previous studies have shown the successful implementation of DL variant caller tools in biomedical research [35, 36], and that they can outperform existing state-of-the-art tools [36]. Other DL models have been used for gene conservation and expression analysis, CRISPRCas9 off-target prediction and protein analysis [37-42]. The training of a DL model requires a massive amount of processed and annotated data, which has restricted its application in plant 
pangenome assembly and analysis for several applications, since the model needs accurately labelled samples in order to learn the features of the target. In other domains, DL models can integrate a previously highly trained model as an initial step of learning, which greatly accelerates the training and decreases the initial data required [43]. This step is not currently available for genomic data models, but the increasing availability of high quality annotations in plant sequencing data should soon address this limitation.

There are a wide array of potential applications for DL algorithms in plant pangenome analysis as they require few if any predefined assumptions, allowing for unbiased analysis. The high complexity of plant pangenomes can be leveraged by the DL models, which are able to recognise the underlying features within species or cross-species genomes, allowing for the identification of the mechanisms that lead to genome variability. The ongoing increase of functional annotations in plant genomes provide the foundation for the training of new DL tools capable of predicting sequence function with increasing accuracy. The potential of DL to integrate multi-omics data with sequence data supports a multidimensional characterisation of the plant pangenome. These trained DL models could then become a powerful tool for breeding and evolutionary analysis by generating accurate association of genes with agronomic traits.

\section{Pangenome visualisation}

The visualisation of the genomic relationships within the pangenome is important for the interpretation of conserved and variable genomic features. Visualisation tools have been developed for microbial pangenomes [44], and whilst some can be adapted to plants, it has proven difficult to design scalable solutions for large panels of samples. Most of the visualisation tools developed for pangenomes are either based on a reference genome structure (building the dispensable region based on gene ontology and PAV patterns), or require a de novo assembly with VG in order to properly display the dispensable regions and pathways (Figure 3; [26, 45, 46]). For example, ppsPCP and CoGe use assembled plant genome comparisons, enabling the visualisation of PAVs and other variable regions $[47,48]$. Some plant pangenomes studies have released tools tailored for the visualisation of their data, such as RPAN with 3000 rice genomes, GBrowse with 16 wheat cultivars, and Brachypan using 54 Brachypodium distachyon lines [9, 16, 49]. These tools allow searching for genes and visualisation within the pangenome databases, providing access to genomic sequences, gene annotations, PAV information and gene expression information. The development of robust visualisation tools for other species is 
necessary to facilitate detailed integrated analysis and support pangenome applications for crop improvement.

The complexity, variability and size of plant genomes present a significant computational challenge for the development of pangenome visualisation tools. To overcome this limitation, some pangenome researchers have embraced cloud-based solutions to facilitate the accessibility of tools for pangenome manipulation and visualisation [50-52]. The cloud here is defined as an infrastructure that provides services through the internet. Cloud databases provide data storage and curation as well as software maintenance in high performance computers, increasing reproducibility. A cloud "commons" environment, such as that developed for biomedical collaboration [50,53], could facilitate the integration of plant multi-omics and auxiliary data such as geographic, phenotypic, gene expression and functional data in a common database. The establishment of a cloud based hub for plant pangenomes could also facilitate data and analytics sharing between researchers, enabling collaborative pangenome characterisation and supporting the establishment and use of common standards and approaches.

\section{Application of pangenomes for crop improvement}

Genomic information is increasingly being applied within crop breeding, however reference genome assemblies may not be representative of the germplasm in the field [16], emphasizing the need for pangenomes in crop improvement applications. Pangenomes can be used to map the dispensable genome, highlighting which varieties host genes that provide an agronomic benefit. The dispensable genome of crops is often associated with agronomic traits such as flowering time and disease resistance [1, 2, 14, 54] and environmental stress response [55-57]. A reduction in genetic variation has been observed during the breeding of some crops, particularly associated with the selection of important traits such as disease resistance [58, 59]. The development of pangenomes supports the characterisation of gene PAV within crop species, as well as comparison with wild and exotic relatives. This process can identify genes that have been lost during breeding and selection which may confer positive agronomic attributes that can be bred back into elite germplasm for evaluation.

The identification of dispensable regions in a species advances the understanding of which genomic variations may be associated with agronomic performance. Associations have improved through the use of high throughput molecular genetic markers such as SNPs, and the 
application of pangenome wide SNPs as well as the inclusion of PAV in the association analysis, increases the power of genomic associations and the identification of causal variants for agronomic traits [13].

Developments in the field of genome editing such as CRISPR-Cas [60] have the potential to revolutionise crop breeding approaches, moving from traditional crossing and selection towards rounds of genome editing and selection [61-63]. Knowledge of the full complement of the gene content is required for applied genome editing based breeding, both to ensure that the correct target is present in the genome to be modified, and importantly to avoid off target effects where related sequence are accidently also edited, with unpredictable consequences [64].

\section{Cross-species pangenomes and evolutionary studies}

The majority of pangenomes constructed to date have been for a single species, however there is the potential for the production of pangenomes representing multiple related species representing higher taxonomic groups. This can assist in the identification of genes that are specific to individual species as well as those which are common across broader taxonomic groups. This provides biologists with the tools to explore what defines each species from a genomic perspective, which in some cases may lead to the identification of taxonomic inconsistencies that can then be resolved, as it has been done in bacterial pangenomics [65].

Plant genomes are highly dynamic, with gene-size insertions leading to the amplification of gene families and the generating of new genes through gene fusions, so the characterisation of SV in core and dispensable genes can provide a valuable approach to understand gene evolution [66]. Pangenomes support analysis of the evolutionary history of genes, including the identification of gene orthology and an understanding of gene family features. This information is important for understanding gene function and can provide boundaries for our ability to translate function from one gene/species to another. Knowledge of pangenome evolution can also support the more accurate automated annotation of genomes through a greater understanding of gene sequence diversity. Gene processes such as directional selection, divergence and neofunctionalisation can then be linked to phenotypic traits with greater accuracy [44]. Genome-wide patterns can also be explored using gene content from pangenomes and by using pangenomic data to map the evolutionary divergence that has taken place in different lineages [12], enabling researchers to explore the genomic basis of adaptations associated with ecological niches. 


\section{Challenges and future perspectives with Pangenomics}

The construction of pangenomes, both within and across species, is becoming increasingly attractive, as large scale collaborative efforts such as the "10KP Genome Sequencing Project" [67] expand the availability of sequence data from a wide range of plants. However, future research requires new tools to support variation graph assembly, pangenome construction and visualisation.

While pangenomes have the potential to increase the efficiency of crop breeding, future tools and databases must integrate pangenomes with gene functional analyses and evolutionary reconstruction. A comprehensive set of pangenome tools, capable of integrating diverse 'omics data is needed to unravel the relationships between genomic variation (including non-coding regions) and complex agronomic traits. As crop gene editing technologies continue to evolve and become more widespread [68], in part due to relatively permissive regulatory environment compared to GM crops [69], there will be a need to expand the germplasm pool and our understanding of the broader genomic landscape of crop species, providing the template for genome editing based crop improvement.

Several challenges remain to be addressed to enable the cost effective and rapid construction of accurate pangenomes and their integration with available functional data. The integration of long sequence reads can assist in resolving repetitive motifs and polyploid genomes which prove difficult to assemble with short read sequences, and this approach is likely to dominate plant pangenomics once as the accuracy of this data increases, with decreasing costs of data production [70, 71]. The application of deep learning in pangenomics may also aid in overcoming some challenges of pangenome assembly and functional characterisation due to its ability to autonomously identify patterns in the data [34].

Whilst many of the major crops now have pangenomes available, with variable levels of completeness, expanding these beyond their species will enable increased use of wild gene sequence diversity in crop improvement, supporting the development of designer crops. Once pangenomes are available for a large number of diverse species, we can start to understand how species and higher taxa are defined at the genome level, providing deep insights into plant evolution and diversification. 


\section{Acknowledgements}

This work was supported by the Australian Government through the Australian Research Council (Projects LP160100030, LP140100537 and LP130100925) and the award of Research Training Program scholarships to Cassandria T. Fernandez, Jacob Marsh and Monica F. Danilevicz. Monica F. Danilevicz was also supported by the Forrest Research Foundation. The views and opinions of authors expressed herein do not necessarily state or reflect those of the funding organisations as they had no involvement in the design or production of the study. We would like to thank Felipe Casaprima for the graphic design of the images used in this review.

\section{Competing interests}

The authors declare that they have no known competing financial interests or personal relationships that could have appeared to influence the work reported in this paper.

1. The Rice, C. and C. Sequencing, The sequence of rice chromosomes 11 and 12, rich in disease resistance genes and recent gene duplications. BMC Biology, 2005. 3(1): p. 20.

2. $\mathrm{Yu}, \mathrm{J}$., et al., Genome-wide comparative analysis of NBS-encoding genes between Brassica species and Arabidopsis thaliana. BMC Genomics, 2014. 15(1): p. 3.

3. Chen, J.-Y., et al., Genome-wide analysis of the gene families of resistance gene analogues in cotton and their response to Verticillium wilt. BMC Plant Biology, 2015. 15(1): p. 148.

4. Bennetzen, J.L., Transposable element contributions to plant gene and genome evolution. Plant Molecular Biology, 2000. 42(1): p. 251-269.

5. McClintock, B. Controlling elements and the gene. in Cold Spring Harbor symposia on quantitative biology. 1956. Cold Spring Harbor Laboratory Press.

6. Gabur, I., et al., Connecting genome structural variation with complex traits in crop plants. Theoretical and Applied Genetics, 2019. 132(3): p. 733-750.

7. Claros, M.G., et al., Why assembling plant genome sequences is so challenging. Biology, 2012. 1(2): p. 439-459.

8. Lu, F., et al., High-resolution genetic mapping of maize pan-genome sequence anchors. Nature Communications, 2015. 6: p. 6914.

9. Gordon, S.P., et al., Extensive gene content variation in the Brachypodium distachyon pan-genome correlates with population structure. Nature Communications, 2017. 8(1): p. 2184.

10. Li, Y.-h., et al., De novo assembly of soybean wild relatives for pan-genome analysis of diversity and agronomic traits. Nature Biotechnology, 2014. 32: p. 1045.

11. Schatz, M.C., et al., Whole genome de novo assemblies of three divergent strains of rice, Oryza sativa, document novel gene space of aus and indica. Genome biology, 2014. 15(11): p. 506.

12. Zhao, Q., et al., Pan-genome analysis highlights the extent of genomic variation in cultivated and wild rice. Nature Genetics, 2018. 50(2): p. 278-284.

13. Hurgobin, B. and D. Edwards, SNP discovery using a pangenome: has the single reference approach become obsolete? Biology, 2017. 6(1): p. 21. 
14. Golicz, A.A., et al., The pangenome of an agronomically important crop plant Brassica oleracea. Nature Communications, 2016. 7: p. 13390.

15. Hübner, S., et al., Sunflower pan-genome analysis shows that hybridization altered gene content and disease resistance. Nature plants, 2019. 5(1): p. 54.

16. Montenegro, J.D., et al., The pangenome of hexaploid bread wheat. The Plant Journal, 2017. 90(5): p. 1007-1013.

17. Zhang, B., et al., The poplar pangenome provides insights into the evolutionary history of the genus. Communications Biology, 2019. 2(1): p. 215.

18. Jiao, W.-B. and K. Schneeberger, The impact of third generation genomic technologies on plant genome assembly. Current Opinion in Plant Biology, 2017. 36: p. 64-70.

19. Tao, Y., et al., Exploring and Exploiting Pan-genomics for Crop Improvement. Molecular Plant, 2019. 12(2): p. 156-169.

20. Goodwin, S., J.D. McPherson, and W.R. McCombie, Coming of age: ten years of nextgeneration sequencing technologies. Nature Reviews Genetics, 2016. 17(6): p. 333.

21. Lan, T., et al., Long-read sequencing uncovers the adaptive topography of a carnivorous plant genome. Proceedings of the National Academy of Sciences, 2017. 114(22): p. E4435.

22. Paajanen, P., et al., A critical comparison of technologies for a plant genome sequencing project. GigaScience, 2019. 8(3).

23. Schmidt, M.H.W., et al., De Novo Assembly of a New \&lt;em\&gt;Solanum pennellii\&lt;/em\&gt; Accession Using Nanopore Sequencing. The Plant Cell, 2017. 29(10): p. 2336.

24. Garrison, E., et al., Variation graph toolkit improves read mapping by representing genetic variation in the reference. Nature biotechnology, 2018.

25. Rakocevic, G., et al., Fast and accurate genomic analyses using genome graphs. Nature Genetics, 2019. 51(2): p. 354-362.

26. Eggertsson, H.P., et al., Graphtyper enables population-scale genotyping using pangenome graphs. Nature Genetics, 2017. 49: p. 1654.

27. Jackman, S.D., et al., ABySS 2.0: resource-efficient assembly of large genomes using a Bloom filter. Genome research, 2017. 27(5): p. 768-777.

28. Liao, C.-C., et al., EAT-Rice: A predictive model for flanking gene expression of T-DNA insertion activation-tagged rice mutants by machine learning approaches. PLOS Computational Biology, 2019. 15(5): p. e1006942.

29. Simopoulos, C.M.A., E.A. Weretilnyk, and G.B. Golding, Prediction of plant IncRNA by ensemble machine learning classifiers. BMC Genomics, 2018. 19(1): p. 316.

30. Zhai, J., et al., PEA: an integrated $R$ toolkit for plant epitranscriptome analysis. Bioinformatics, 2018. 34(21): p. 3747-3749.

31. Zhang, N., et al., MU-LOC: A Machine-Learning Method for Predicting Mitochondrially Localized Proteins in Plants. Frontiers in Plant Science, 2018. 9(634).

32. Eraslan, G., et al., Deep learning: new computational modelling techniques for genomics. Nature Reviews Genetics, 2019. 20(7): p. 389-403.

33. Libbrecht, M.W. and W.S. Noble, Machine learning applications in genetics and genomics. Nature Reviews Genetics, 2015. 16: p. 321.

34. LeCun, Y., Y. Bengio, and G. Hinton, Deep learning. Nature, 2015. 521: p. 436.

35. Cai, L., Y. Wu, and J. Gao, DeepSV: Accurate calling of genomic deletions from high throughput sequencing data using deep convolutional neural network. bioRxiv, 2019: p. 561357.

36. Poplin, R., et al., A universal SNP and small-indel variant caller using deep neural networks. Nature Biotechnology, 2018. 36: p. 983.

37. Almagro Armenteros, J.J., et al., DeepLoc: prediction of protein subcellular localization using deep learning. Bioinformatics, 2017. 33(21): p. 3387-3395. 
38. Angermueller, C., et al., DeepCpG: accurate prediction of single-cell DNA methylation states using deep learning. Genome biology, 2017. 18(1): p. 67.

39. Chen, L., A.E. Fish, and J.A. Capra, Prediction of gene regulatory enhancers across species reveals evolutionarily conserved sequence properties. PLOS Computational Biology, 2018. 14(10): p. e1006484.

40. Chen, Y., et al., Gene expression inference with deep learning. Bioinformatics, 2016. 32(12): p. 1832-1839.

41. Hashemifar, S., et al., Predicting protein-protein interactions through sequence-based deep learning. Bioinformatics, 2018. 34(17): p. i802-i810.

42. Wang, J., et al., Computational Protein Design with Deep Learning Neural Networks. Scientific Reports, 2018. 8(1): p. 6349.

43. He, K., et al. Deep residual learning for image recognition. in Proceedings of the IEEE conference on computer vision and pattern recognition. 2016.

44. Ding, W., F. Baumdicker, and R.A. Neher, panX: pan-genome analysis and exploration. Nucleic acids research, 2017. 46(1): p. e5-e5.

45. Hennig, A., J. Bernhardt, and K. Nieselt, Pan-Tetris: an interactive visualisation for Pangenomes. BMC Bioinformatics, 2015. 16(11): p. S3.

46. Pedersen, T.L., et al., PanViz: interactive visualization of the structure of functionally annotated pangenomes. Bioinformatics, 2016. 33(7): p. 1081-1082.

47. Tahir UI Qamar, M., et al., ppsPCP: a plant presence/absence variants scanner and pan-genome construction pipeline. Bioinformatics, 2019. 35(20): p. 4156-4158.

48. Lyons, E. and M. Freeling, How to usefully compare homologous plant genes and chromosomes as DNA sequences. The Plant Journal, 2008. 53(4): p. 661-673.

49. Sun, C., et al., RPAN: rice pan-genome browser for 3000 rice genomes. Nucleic acids research, 2016. 45(2): p. 597-605.

50. Connor, T.R., et al., CLIMB (the Cloud Infrastructure for Microbial Bioinformatics): an online resource for the medical microbiology community. Microbial genomics, 2016. 2(9).

51. Jagodnik, K.M., et al., Developing a framework for digital objects in the Big Data to Knowledge (BD2K) commons: Report from the Commons Framework Pilots workshop. Journal of biomedical informatics, 2017. 71: p. 49-57.

52. Navale, V. and P.E. Bourne, Cloud computing applications for biomedical science: A perspective. PLoS computational biology, 2018. 14(6): p. e1006144.

53. Brody, J.A., et al., Analysis commons, a team approach to discovery in a big-data environment for genetic epidemiology. Nature genetics, 2017. 49(11): p. 1560.

54. Bayer, P.E., et al., Variation in abundance of predicted resistance genes in the Brassica oleracea pangenome. Plant Biotechnology Journal, 2019. 17(4): p. 789-800.

55. Hardigan, M.A., et al., Genome Reduction Uncovers a Large Dispensable Genome and Adaptive Role for Copy Number Variation in Asexually Propagated \&lt;em\&gt;Solanum tuberosum\&lt;/em\&gt. The Plant Cell, 2016. 28(2): p. 388.

56. $\mathrm{Xu}, \mathrm{K}$., et al., Sub1A is an ethylene-response-factor-like gene that confers submergence tolerance to rice. Nature, 2006. 442(7103): p. 705-708.

57. Hoopes, G.M., et al., An updated gene atlas for maize reveals organ-specific and stressinduced genes. The Plant Journal, 2019. 97(6): p. 1154-1167.

58. Krattinger, S.G. and B. Keller, Molecular genetics and evolution of disease resistance in cereals. New Phytologist, 2016. 212(2): p. 320-332.

59. Zhou, Z., et al., Resequencing 302 wild and cultivated accessions identifies genes related to domestication and improvement in soybean. Nature biotechnology, 2015. 33(4): p. 408.

60. Cong, L., et al., Multiplex Genome Engineering Using CRISPR/Cas Systems. Science, 2013. 339(6121): p. 819. 
61. Scheben, A. and D. Edwards, Genome editors take on crops. Science, 2017. 355(6330): p. $1122-1123$.

62. Scheben, A. and D. Edwards, Bottlenecks for genome-edited crops on the road from lab to farm. Genome Biology, 2018. 19(1): p. 178.

63. Scheben, A., et al., Towards CRISPR/Cas crops - bringing together genomics and genome editing. New Phytologist, 2017. 216(3): p. 682-698.

64. Zhang, X.-H., et al., Off-target effects in CRISPR/Cas9-mediated genome engineering. Molecular Therapy-Nucleic Acids, 2015. 4: p. e264.

65. Wuyts, S., et al., Large-scale phylogenomics of the Lactobacillus casei group highlights taxonomic inconsistencies and reveals novel clade-associated features. MSystems, 2017. 2(4): p. e00061-17.

66. Krasileva, K.V., The role of transposable elements and DNA damage repair mechanisms in gene duplications and gene fusions in plant genomes. Current Opinion in Plant Biology, 2019. 48: p. 18-25.

67. Cheng, S., et al., 10KP: A phylodiverse genome sequencing plan. Gigascience, 2018. 7(3): p. giy013.

68. Wang, T., H. Zhang, and H. Zhu, CRISPR technology is revolutionizing the improvement of tomato and other fruit crops. Horticulture research, 2019. 6(1): p. 77.

69. Waltz, E., With a free pass, CRISPR-edited plants reach market in record time. 2018, Nature Publishing Group.

70. Berlin, K., et al., Assembling large genomes with single-molecule sequencing and locality-sensitive hashing. Nature biotechnology, 2015. 33(6): p. 623.

71. Jung, H., et al., Tools and Strategies for Long-Read Sequencing and De Novo Assembly of Plant Genomes. Trends in Plant Science, 2019.

Highlights

- Pan-genomes represent the complex genomic diversity found in plant species

- Novel approaches are under development to enable more complex pan-genome assemblies

- Integration of auxiliary data with pan-genomes provides insights into gene evolution

- Pan-genomes of crops and related species enable the identification of novel alleles

- New tools are required to analyse complex pan-genomes and associated data 


\section{Annotated References}

Garrison et al., 2018**

VG is a variation-graph assembler tool is presented for the creation of bi-directional graph representations of eukaryotic pan-genomes, which have several advantages over linear pangenomes. VG enables the creation, manipulation and visualisation of the graph pan-genome, allowing for a more accurate and complete read mapping (tested at human genome size).

Poplin et al., 2018**

This study describes a tool for SNP calling using deep convolutional neural networks to analyse aligned sequence data. The DeepVariant relies exclusively on autonomously extracted features for the identification of genetic variants. DeepVariant appears to be robust to changes in sequencing depth, preparation protocol, instrument type and genome build, with the ability to utilize training data across species.

Zhao et al., 2018*

Pan-genome construction of cultivated and wild rice (Oryza sativa and Oryza rufipogon) from 1,529 accessions. This study showcases pan-genome construction and its use in investigating genetic variation and characterizing novel complex variants that were overlooked in the rice single reference genome.

Krasileva et al., 2019*

This study describes the role of transposable elements and DNA repair machinery in gene-sized insertions, which can cause the amplification of gene families as well as facilitate new gene fusions. These mechanisms generally present specificity towards a gene family, plant lineage or environmental conditions, requiring comparative studies of pangenomes to better understand how structural variants arise.

Jiao et al., 2017*

This review focuses on third generation sequencing technologies, in particular long-range scaffolding. Long-read scaffolding have thus far outperformed short-read scaffold assembly, generally being more accurate and in depth. Long-read sequences have the potential to assemble contigs to a higher degree of detail (chromosomal level) with very little increase in 
sequence costs. The review also details how third generation technologies assists in heterozygous and polyploid assembly.

Wang et al., 2019*

This review article covers the recent history of CRISPR gene editing technology for fruit crop improvement and presents perspectives and controversies surrounding its implementation.

Whilst the article focuses heavily on tomato research since 2014 , it provides a good introduction into the current state of crop gene editing and its different applications. 
Figure 1: Pan-genome assembly approaches: Pan-genome assembly methods from genomes $A-D$, as well as $E$ as an alternative reference for the iterative assembly approach. The sequencing reads (top) are used to reconstruct the original sequence of each genome. The genomes are composed of several genomic segments represented by different colours. Shared genomic segments have the same colour in all genomes. As show in $A$, the de novo assembly approach builds each genome separately, enabling direct comparisons to identify the core and variable regions. The iterative approach $(B)$ uses a single genome as a reference to which nonredundant sequences from other genomes are added. The sequences that cannot be directly added to the reference genome are assembled using the de novo approach before being incorporated into the pan-genome. In this approach, the choice of the reference genome significantly influences the placement of the genomic segments in the pan-genome, as shown by comparing the pan-genome assembly using the genome $A$ as reference, and using the genome $\mathrm{E}$ as reference. In the variation graph approach (C), the pan-genome sequence is assembled using a De Bruijn graph that splits the genome into segments. The relationships between these genomic segments within the pan-genome (i.e. the original genomic sequences) can be traced by following the paths of the graph.

Figure 2: Deep learning models for plant pan-genome analysis: Section A of the figure shows a simplified workflow for developing a supervised DL model. In the model depicted the labelled data is fed into the initial model for training, after a few iterations the model is tested with unlabelled test data. Test data, not used during the training step, is used to assess the performance of the DL algorithm, enabling fine tuning of parameters to improve the model. The functional deep learning algorithm has been formed based on the labelled data and tested with the unlabelled data. Common inputs for genomic DL models (B) include sequence alignment images, nucleotide sequences (e.g. FASTA) and tabular data. The first hidden layers extracts the most simple features, whereas in the last hidden layers the features will be more complex. The last hidden layers utilise information from previous layers to form features that take into account complex patterns found in the training data. In the last layer, the DL model outputs the result of the analysis, which can be a classification, prediction or clustering of the data, as previously defined.

Figure 3: Variation graph pan-genome visualisation: The construction of the pan-genome variation graph creates paths for each genomic region interaction, which can be broadly visualised as shown in $\mathrm{B}$. The nine genomes in this pan-genome graph are represented by 
coloured lines. Genomes from the same population share line colour. Pan-genome visualisation tools allow for close inspection of the variable regions, as shown in A and C. In C it is possible to observe the copy number variation and presence absence variation within and across populations by following the lines. It should be noted that it is possible to zoom in further to observe genomic variation at the nucleotide level (A), showing SNPs and smaller indels. 


\section{Figure 1}

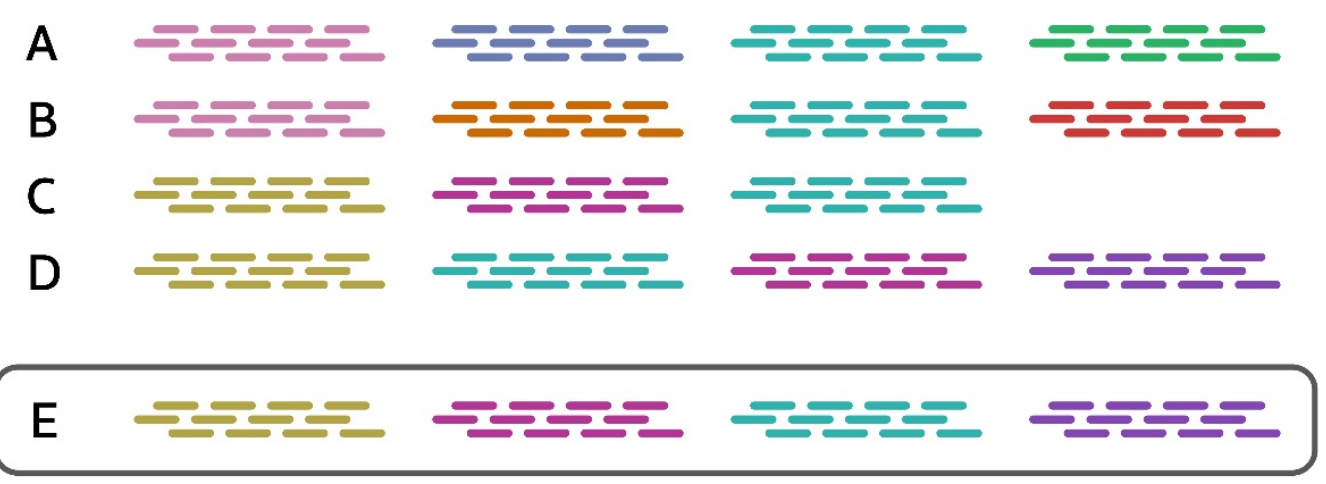

1. De novo assembly

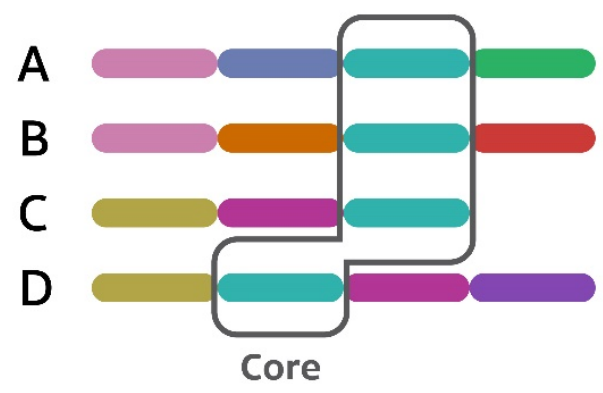

2. Iterative assembly

Using genome A as reference

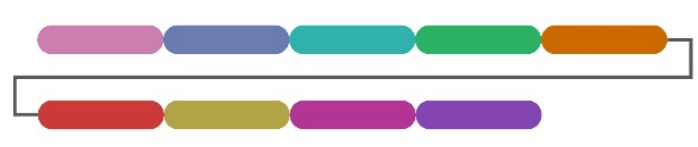

Using genome $E$ as reference

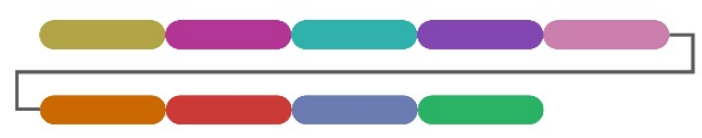

3. Variation graph

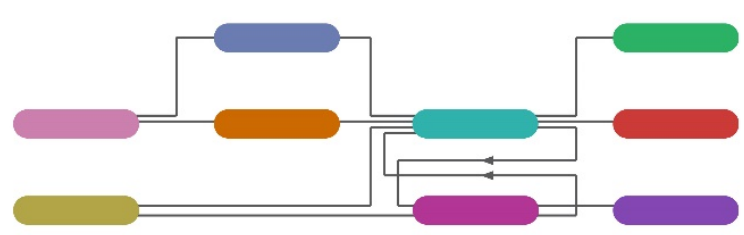




\section{Figure 2}

A

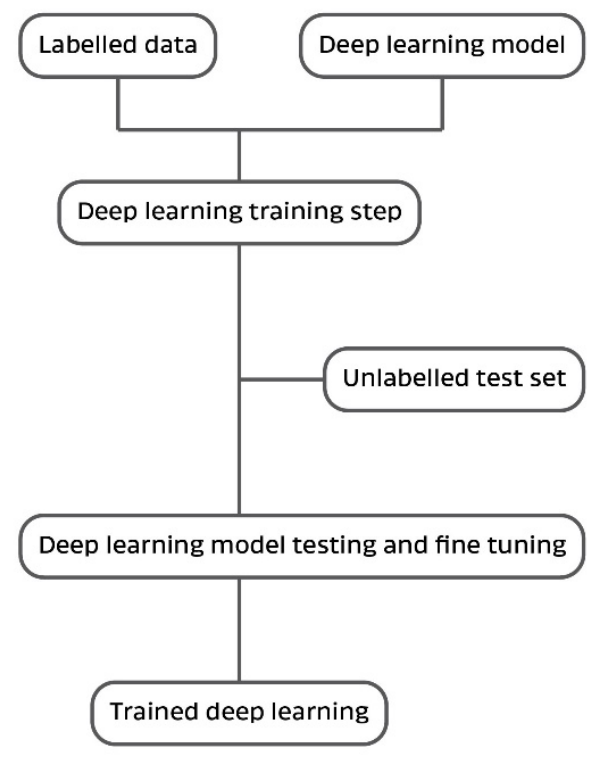

B

\section{Types of Inputs}

Image data

ATGACTAACTACC

ATGACCAACTACC

AT TEACCAACTACC
AT TACCATCCACC

AT
ATGACCATCCACACC

A TOECAAACCACC

ATGACCAACTACC

A TGACCATCCACC

\section{Text data}

ACGTCACGTACTAG

CATGCATCGTAGC

GTACGTACGTAGCT

Tabular data

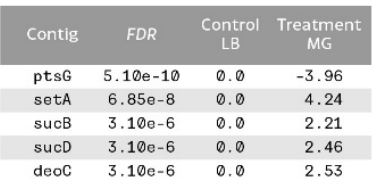

Input Layer

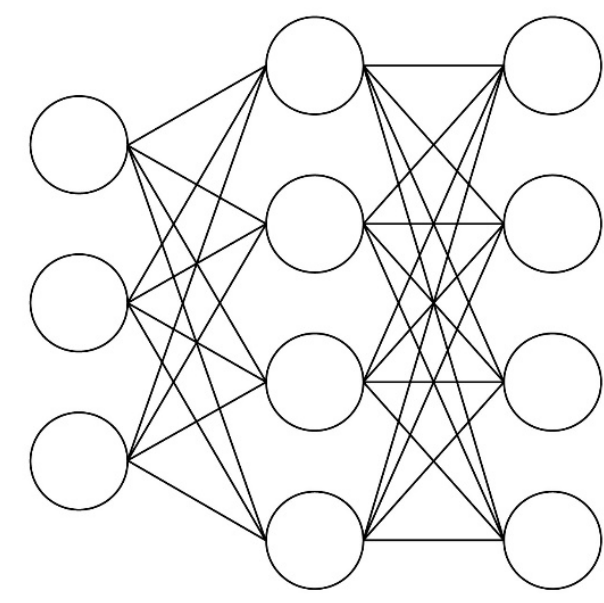

Hidden Layer 1
...

Output Layer

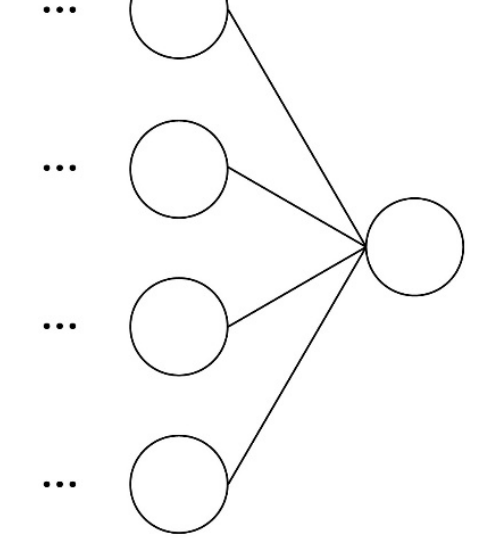

Hidden Layer $n$ 
Figure 3

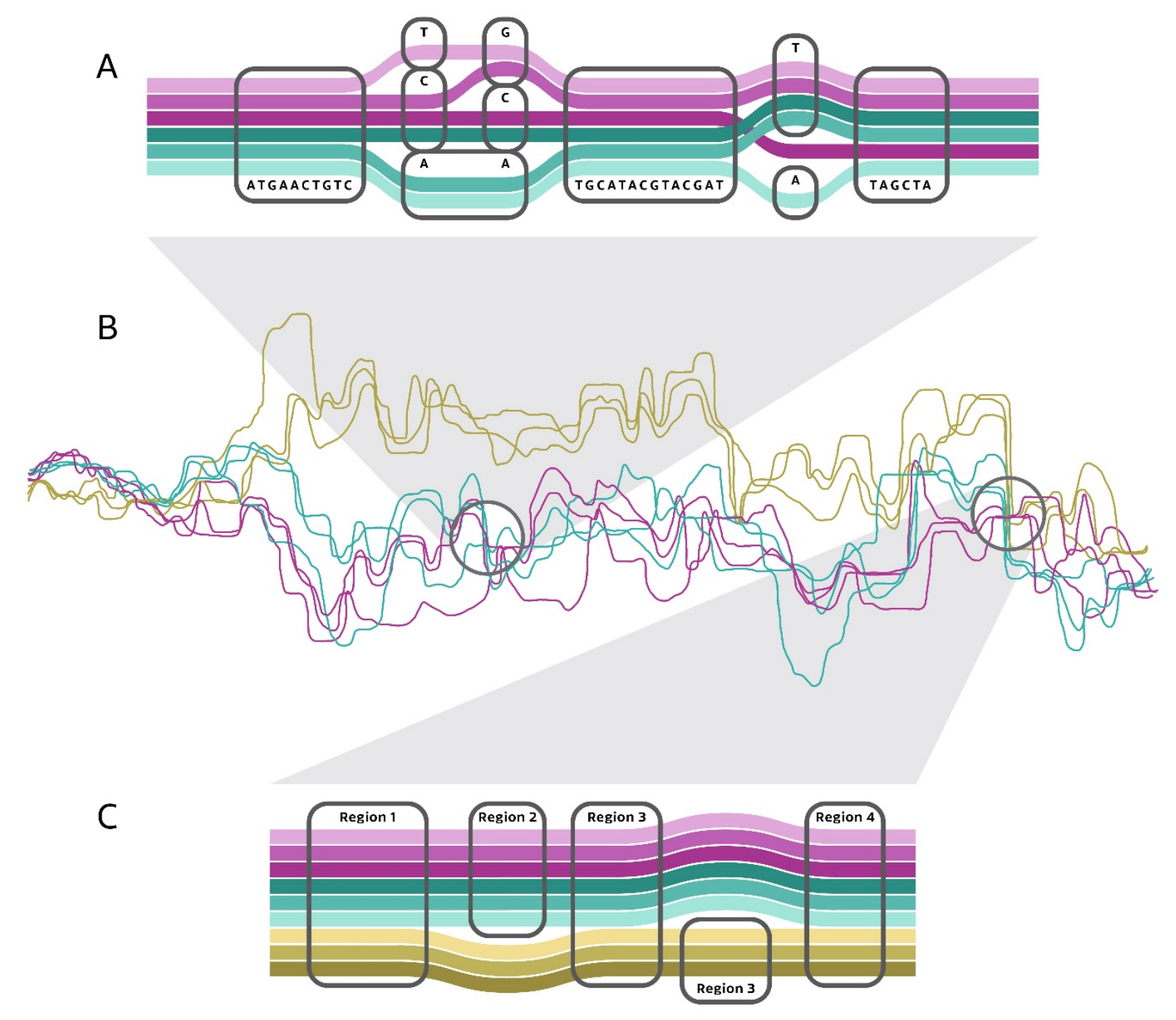

